\title{
SELETIVIDADE DE ALGUNS AGROTÓXICOS EM USO NA CITRICULTURA AO ÁCARO PREDADOR Neoseiulus californicus (McGREGOR) (ACARI: PHYTOSEIIDAE) ${ }^{1}$
}

\author{
MARCOS ZATTI DA SILVA² \& CARLOS AMADEU LEITE DE OLIVEIRA ${ }^{3}$
}

\begin{abstract}
RESUMO - Objetivou-se avaliar o efeito dos principais agrotóxicos utilizados em citros sobre Neoseiulus californicus (McGregor), em condições de laboratório, por tratar-se de um fitoseídeo com grande potencialidade para ser utilizado no controle dos ácaros fitófagos na cultura. O método de bioensaio adotado foi o de contato direto e residual. Folhas de citros da variedade Pêra, contendo 25 fêmeas adultas de $N$. californicus procedentes de uma criação-estoque, foram pulverizadas em torre de Potter. Avaliaram-se a mortalidade do ácaro 72 horas após a aplicação e o efeito dos produtos na viabilidade dos ovos. Quanto à seletividade, conforme proposta da "Organização Internacional para o Controle Biológico e Integrado de Plantas e Animais Nocivos" (IOBC), os produtos foram classificados como: classe 1- inócuo (E<30\%), acrinathrin, deltamethrin, dinocap, enxofre, óxido de fenbutatin e propargite; 2-levemente nocivo (30\%<E<79\%), azocyclotin, cyhexatin e fenpropathrin; 3- moderadamente nocivo ( $80 \%<\mathrm{E}<99 \%)$, abamectin ; 4- nocivo (E>99\%), clorfenapyr, dicofol e pyridaben. $N$. californicus mostrou-se tolerante a diversos agrotóxicos, o que viabiliza sua utilização, juntamente com produtos químicos pertencentes a grupamentos distintos e de diferentes mecanismos de ação, o que atende a um manejo adequado de controle dos principais ácaros fitófagos da cultura dos citros.
\end{abstract}

Termos para indexação: inimigos naturais, citros, manejo integrado de pragas, controle biológico

\section{RESIDUALTOXICITY OFTHEMAIN PESTICIDESRECOMMENDEDINCITRUSORCHARDSONPREDACEOUSMITENeoseiulus californicus (McGREGOR) (ACARI: PHYTOSEIIDAE).}

\begin{abstract}
This work was carried out to determine the residual toxicity of the main pesticides used in citrus, on Neoseiulus californicus (McGregor) under laboratory conditions. For this, it was used the residual contact bioassay. Citrus leaves of the variety "Pêra" were sprayed in a Potter tower. The residual action was evaluated at 2 hours and $1 ; 3 ; 5 ; 7 ; 10 ; 14$ e 21 days after treatment. Ten adult females of $N$. californicus were transferred to each ring, together with an enough amount of Tetranychus urticae nymphs and adults to feed the predator. Mortality evaluations were performed at 72 hours after treatment. Bioassays indicate that the pesticides: acrinathrin, deltamethrin, dinocap, sulphur, fenpropathrin, fenbutatin oxide and propargite were innocuous to $N$. californicus. On the other hand, abamectin, azocyclotin e cyhexatin caused mortalities of $29.8 ; 32.0$ e $34.1 \%$, respectively, two hours after transference. Dicofol, pyridaben and chlorfenapyr were extremely toxic to the predator mite, causing $100 \%$ of mortality two hours after the application. This way, to this population of $N$. californicus, the releases can be performed safely 3 days after the application, except for cyhexatin (5 days), dicofol and pyridaben (14 days) and chlorfenapyr (21 days), without risk of significant adult mortality rates due to the application of pesticides.
\end{abstract}

Index Terms: Predatory mite, citrus, phytoseiid, biological control, sensibility.

\section{INTRODUÇÃO}

A utilização de agrotóxicos sem o conhecimento de sua ação sobre inimigos naturais tem provocado sérios problemas em diversas culturas. Ressurgência e surtos de pragas secundárias são alguns destes problemas enfrentados pelos produtores agrícolas (Van de Vrie et al., 1972). Outro problema associado ao uso excessivo de agrotóxicos é o desenvolvimento da resistência a alguns acaricidas utilizados na cultura (Omoto et al., 2000).

Nos últimos anos, alguns trabalhos foram realizados para avaliar a seletividade de agrotóxicos a ácaros predadores da família Phytoseiidae presentes em citros, em nossas condições (Sato et al., 1994, 1996; Yamamoto et al., 1992; Reis et al., 1998, 1999). A escolha de produtos seletivos é indispensável para minimizar os efeitos prejudiciais sobre a fauna e manter o equilíbrio biológico no agroecossistema (Busoli, 1992).

Dentre os inimigos naturais dos ácaros fitófagos, aqueles pertencentes à família Phytoseiidae são os mais importantes (McMurtry et al., 1970), sendo mais de 1.700 espécies acarinas descritas mundialmente (Kostiainen \& Hoy, 1996), das quais aproximadamente 100 já foram observadas no Brasil (Moraes, 1992).

Neoseiulus californicus (McGregor) é um ácaro fitoseídeo, que promove o controle biológico de tetraniquídeos em diversas plantas cultivadas, como morangueiro, macieira, feijoeiro, ornamentais, etc. Ocorre nas regiões semitropicais e temperadas da América do Sul, e também nas áreas áridas do sul da Califórnia e sul da Europa (McMurtry \& Croft, 1997).

No Brasil, N. californicus tem sido criado massalmente e liberado em pomares de macieira no sul do País, visando ao controle de Panonychus ulmi Koch, o qual tem se mostrado um inimigo natural bastante efetivo, contribuindo para uma redução significativa no uso de acaricidas (Monteiro, 1994).

A integração do controle químico e biológico no manejo de ácaros fitófagos pode ser uma alternativa para um efetivo controle desses organismos. A utilização de $N$. californicus em programas de controle biológico em citros é promissora, haja vista o grande número de ácaros fitófagos presentes na cultura, razão pela qual se objetiva, no presente trabalho, avaliar a seletividade de alguns dos principais agrotóxicos a $N$. californicus, em sua maioria acaricidas, utilizados em citros.

\section{MATERIAL E MÉTODOS}

A pesquisa foi realizada no Laboratório de Acarologia do Departamento de Fitossanidade da Faculdade de Ciências Agrárias e Veterinária, da Universidade Estadual Paulista, Câmpus de Jaboticabal - SP.

Criação de Neoseiulus californicus - Iniciou-se uma criaçãoestoque de $N$. californicus, a partir de uma população cedida pelo Dr. Mário Eidi Sato do Laboratório de Entomologia Econômica do Centro Experimental Central, do Instituto Biológico, Campinas - SP, coletada em cultivo comercial de morangueiro, no município de Atibaia - SP, em 30-10-1999. Da criação-estoque, mantida no laboratório sobre plantas de feijão-de-porco [Canavalia ensiformis (L.)] envasadas, tendo como fonte alimentar Tetranychus urticae Koch, foram transferidos ácaros para discos de folha de laranjeira da variedade Pêra, com aproximadamente $8 \mathrm{~cm}$ de diâmetro. Os discos foram colocados sobre

\footnotetext{
${ }^{1}$ (Trabalho 060-2006). Recebido: 08-05-2006. Aceito para publicação: 17-07-2006.

${ }^{2}$ Pós-Graduando em Agronomia-Entomologia Agrícola, FCAV-UNESP. makdsil@ @ig.com.br.

${ }^{3}$ Prof. Titular Departamento de Fitossanidade, amadeu@fcav.unesp.br. Faculdade de Ciências Agrárias e Veterinária-UNESP, Via de acesso Donato Castellane s/n, CEP: 14884-900. Jaboticabal-SP.
} 
TABELA 1 - Relação dos tratamentos estabelecidos no bioensaio.

\begin{tabular}{|c|c|c|c|c|c|}
\hline Produto técnico & $\begin{array}{l}\text { Produto Comercial / } \\
\text { Formulação }\end{array}$ & Uso & $\begin{array}{l}\text { Dosagem (produto } \\
\text { comercial em } 100 \mathrm{~L} \text { ) }\end{array}$ & $\begin{array}{l}\text { Dosagem } \\
\text { (i.a./100L) }\end{array}$ & $\begin{array}{c}\text { Classe } \\
\text { toxicológica }\end{array}$ \\
\hline abamectin & Vertimec CE 18 & $\mathrm{~A}^{1}$ & $30,0 \mathrm{ml}$ & $0,54 \mathrm{~g}$ & III \\
\hline acrinathrin & Rufast 50 SC & $\mathrm{A}$ & $10,0 \mathrm{ml}$ & $0,50 \mathrm{~g}$ & IV \\
\hline azocyclotin & Caligur $500 \mathrm{SC}$ & A & $50,0 \mathrm{ml}$ & $25,00 \mathrm{~g}$ & II \\
\hline cyhexatin & Sipcatin $500 \mathrm{SC}$ & A & $50,0 \mathrm{ml}$ & $25,00 \mathrm{~g}$ & III \\
\hline chlorfenapyr & Citrex $240 \mathrm{SC}$ & $\mathrm{I}, \mathrm{A}$ & $62,5 \mathrm{ml}$ & $15,00 \mathrm{~g}$ & II \\
\hline deltamethrin & Decis $25 \mathrm{CE}$ & I & $50,0 \mathrm{ml}$ & $1,25 \mathrm{~g}$ & II \\
\hline dicofol & Kelthane $480 \mathrm{CE}$ & A & $77,0 \mathrm{ml}$ & $37,00 \mathrm{~g}$ & II \\
\hline dinocap & Karathane $369 \mathrm{CE}$ & $\mathrm{A}$ & $50,0 \mathrm{ml}$ & $18,45 \mathrm{~g}$ & II \\
\hline enxofre & Thiovit 80 PM & $\mathrm{F}, \mathrm{A}$ & $500,0 \mathrm{~g}$ & $400,00 \mathrm{~g}$ & IV \\
\hline fenpropathrin & Danimen $300 \mathrm{CE}$ & $\mathrm{I}, \mathrm{A}$ & $50,0 \mathrm{ml}$ & $15,00 \mathrm{~g}$ & I \\
\hline óxido de fenbutatin & Torque $500 \mathrm{SC}$ & A & $80,0 \mathrm{ml}$ & $40,00 \mathrm{~g}$ & III \\
\hline propargite & Omite $720 \mathrm{CE}$ & $\mathrm{A}$ & $100,0 \mathrm{ml}$ & $72,00 \mathrm{~g}$ & III \\
\hline pyridaben & Sanmite $200 \mathrm{CE}$ & $\mathrm{A}$ & $75,0 \mathrm{ml}$ & $15,00 \mathrm{~g}$ & $\mathrm{I}$ \\
\hline
\end{tabular}

${ }^{1} \mathrm{~A}=$ acaricida; $\mathrm{F}$ = fungicida; $\mathrm{I}=$ inseticida

camada de algodão hidrófilo mantida saturada com água destilada em placa de Petri ( $15 \mathrm{~cm}$ de diâmetro). Para evitar a fuga de ácaros, mantevese a borda da folha coberta com algodão umedecido. Ninfas e adultos de T. urticae e pólen de mamona, Ricinus communis L., foram colocados em abundância em cada arena para servir de alimento aos ácaros predadores, adotando-se metodologia semelhante à de Knight et al. (1990).

Delineamento experimental - O bioensaio foi delineado experimentalmente em parcelas inteiramente casualizadas, onde 13 tratamentos foram repetidos 5 vezes. Cada unidade experimental foi constituída de uma placa de Petri contendo um disco de folha de laranjeira. Os agrotóxicos testados foram empregados nas concentrações recomendadas, segundo Andrei (1999), para o controle do ácaro da leprose Brevipalpus phoenicis, e o deltamethrin (inseticida), na concentração de recomendação para as demais pragas dos citros, não-ácaros (Tabela1). O bioensaio foi conduzido em câmara climatizada a $25 \pm 2^{\circ} \mathrm{C}, 70 \pm 10 \%$ de UR e fotofase de 12 horas.

Adotou-se o método de contato direto e residual para avaliar a seletividade dos produtos. Folhas de citros da variedade Pêra, contendo 25 fêmeas adultas de $N$. californicus, foram pulverizadas em torre de Potter (Burkard Scientific, Uxbridge, UK), com um volume de calda de $2 \mathrm{ml}$, à pressão de $5 \mathrm{lbf} / \mathrm{pol}^{2}$, sobre um disco foliar de laranjeira de $4 \mathrm{~cm}$ de diâmetro, colocado sobre uma camada de algodão hidrófilo, em uma placa de Petri de $9 \mathrm{~cm}$ de diâmetro (Knight et al.,1990).

Critérios de avaliação - As avaliações do número de ácaros vivos e mortos foram realizadas a partir de 24 horas e até 72 horas da aplicação, com auxílio de um microscópio estereoscópico. Consideraram-se mortos os ácaros predadores que não conseguiam locomover-se por uma distância mínima equivalente ao comprimento do seu corpo, ao serem tocados com um pincel de pêlo macio.

Os dados relativos à mortalidade de $N$. californicus foram transformados em $\sqrt{x+0,5}$ e submetidos à analise de variância, pelo teste F, e as médias, comparadas pelo teste Tukey, a 5\% de probabilidade. Os percentuais de eficiência foram calculados segundo a fórmula de Henderson \& Tilton (1955).

O efeito na reprodução $\left(\mathrm{E}_{\mathrm{r}}\right)$ foi calculado dividindo a produção média de ovos no tratamento $\left(\mathrm{R}_{\mathrm{trat}}\right)$ pela produção média de ovos na testemunha $\left(\mathrm{R}_{\text {test }}\right)$, ou seja, $\left(\mathrm{E}_{\mathrm{r}}=\mathrm{R}_{\text {trat. }} / \mathrm{R}_{\text {test }}\right)$. A produção média de ovos por fêmea $(\mathrm{R})$ foi obtida através da relação: $\mathrm{R}=$ total de ovos viáveis/número de fêmeas vivas no final do teste. Foram considerados válidos somente os testes em que a mortalidade na testemunha foi de, no máximo, 20\% (Bakker et al., 1992).

$\mathrm{O}$ efeito total ou adverso (E\%) do produto foi calculado levando-se em conta a mortalidade das fêmeas no tratamento, corrigida em função da mortalidade na testemunha, e o efeito na reprodução, através da fórmula: $\mathrm{E} \%=100 \%-\left(100 \%-\mathrm{M}_{\mathrm{c}}\right) \mathrm{E}_{\mathrm{r}}$, onde $\mathrm{M}_{\mathrm{c}}=$ mortalidade corrigida (Henderson \& Tilton, 1955) e $\mathrm{E}_{\mathrm{r}}=$ efeito na reprodução
(Bakker et al., 1992; Overmeer, 1988; Hassan et al., 1985).

A avaliação da viabilidade dos ovos postos no intervalo de tempo compreendido entre a aplicação e até 72 horas após estendeuse por um período de 7 dias, tempo suficiente para a eclosão das larvas nas condições do bioensaio.

Para a determinação do efeito do agrotóxico sobre $N$. californicus, considerou-se a mortalidade avaliada 72 horas após a aplicação.

Os valores dos efeitos totais obtidos para cada produto foram classificados conforme critérios estabelecidos pela IOBC/WPRS, específicos para organismos benéficos em testes de laboratório (Hassan et al., 1994; Bakker et al., 1992), sendo classe 1: E <30\% (inócuo, não-nocivo); classe 2: 30\%< E <79\% (levemente nocivo); classe $3: 79 \%<\mathrm{E}<99 \%$ (moderadamente nocivo), e classe 4: $\mathrm{E}>99 \%$ ( nocivo ).

\section{RESULTADOS E DISCUSSÃO}

Os resultados dos efeitos dos agrotóxicos sobre o ácaro predador, expressos em mortalidade corrigida $(\mathrm{Mc})$, percentagem de sobrevivência (100 - Mc), efeito na reprodução (Er), efeito total (E\%) e classes de toxicidade a $N$. californicus, são apresentados na Tabela 2.

Os agrotóxicos chlorfenapyr, dicofol e pyridaben mostraramse nocivos a $N$. californicus, causando $100 \%$ de mortalidade dos ácaros (Tabela 2). Esta mortalidade foi observada já nas primeiras 24 horas, demonstrando um forte efeito de choque. Resultados semelhantes foram constatados com Euseius sp. e Iphiseiodes zuluagai (DenmarK \& Muma), principais fitoseídeos em citros e também com N. californicus (Sato et al., 2002; Reis \& Sousa, 2001; Reis et al., 1999; Reis et al., 1998; Santos \& Gravena, 1997).

$\mathrm{O}$ acaricida abamectin acarretou alta mortalidade do $N$. californicus, $79,6 \%$, todavia inferior estatisticamente aos anteriormente citados (Tabela 2 ). Resultados encontrados na literatura, quanto ao efeito desse produto sobre fitoseídeos, são discordantes. Reis et al. (1998 e 1999) constataram baixa toxicidade do produto a $I$. zuluagai com mortalidade de $28 \%$, e a Euseius alatus, com mortalidade de $37 \%$, sendo considerado como inócuo e levemente nocivo, respectivamente; no entanto, Sato et al. (2002) e Araujo et al. (1989) constataram mortalidades superiores a $90 \%$ sobre $N$. californicus em morangueiro, e Euseius concordis e I. zuluagai em citros, por contato direto com abamectim.

Essa divergência de resultados pode ser explicada pelos diferentes métodos adotados, uma vez que Reis et al. (1998 e 1999) avaliaram a ação residual do produto sobre superfície de vidro, e os demais, incluindo o presente trabalho, ocorreram por efeito direto e 
TABELA 2 - Toxicidade de agrotóxicos sobre fêmeas adultas de Neoseiulus californicus, em folhas de citros da variedade Pêra, em bioensaio de contato direto e residual.

\begin{tabular}{|c|c|c|c|c|c|c|}
\hline Produto técnico & Dosagemg de i.a./100L & $\mathrm{M}_{\mathrm{c}}{ }^{1}$ & Sobreviventes $100 \%-\mathrm{M}_{\mathrm{c}}$ & $\mathrm{E}_{\mathrm{r}}^{2}$ & $\mathrm{E}^{3}$ & Classe $^{4}$ \\
\hline abamectin & 0,54 & 79,60 & 20,40 & 0,76 & 84,50 & 3 \\
\hline acrinathrin & 0,50 & 5,40 & 94,60 & 1,01 & 4,45 & 1 \\
\hline azocyclotin & 25,00 & 64,30 & 35,70 & 1,00 & 64,30 & 2 \\
\hline cyhexatin & 25,00 & 41,00 & 59,00 & 0,98 & 42,18 & 2 \\
\hline chlorfenapyr & 15,00 & 100,00 & 0,00 & 0,00 & 100,00 & 4 \\
\hline deltamethrin & 1,25 & 1,30 & 98,70 & 1,01 & 0,31 & 1 \\
\hline dicofol & 37,00 & 100,00 & 0,00 & 0,00 & 100,00 & 4 \\
\hline dinocap & 18,45 & 5,99 & 94,01 & 0,93 & 12,57 & 1 \\
\hline enxofre & 400,00 & 3,10 & 96,90 & 0,96 & 6,98 & 1 \\
\hline fenpropathrin & 15,00 & 22,50 & 77,50 & 0,82 & 36,45 & 2 \\
\hline óxido de fenbutatin & 40,00 & 2,90 & 97,10 & 1,01 & 1,93 & 1 \\
\hline propargite & 72,00 & 13,00 & 87,00 & 0,85 & 26,05 & 1 \\
\hline pyridaben & 15,00 & 100,00 & 0,00 & 0,00 & 100,00 & 4 \\
\hline
\end{tabular}

${ }^{1}$ Mortalidade corrigida: $\mathrm{M}_{c}$; ${ }^{2}$ Efeito na reprodução: $\mathrm{Er}=\mathrm{R}$ trat. / R test. ${ }^{3}$ Efeito total: $\mathrm{E} \%=100 \%$ -

$(100 \%-\mathrm{Mc}) \mathrm{x} \mathrm{Er} ;{ }^{4}$ Classes de toxicidade segundo IOBC/WPRS.

residual, utilizando substratos vegetais, além de tratar-se de populações distintas.

Azocyclotin e cyhexatin causaram mortalidades de 64,3\% e $41 \%$, respectivamente, à população de $N$. californicus, também consideradas elevadas se comparadas às acarretadas pelos produtos acrinathrin, enxofre, óxido de fenbutatin, deltamethrin, dinocap, propargite e fenpropathrin, que foram inferiores a 23\% (Figura 1).

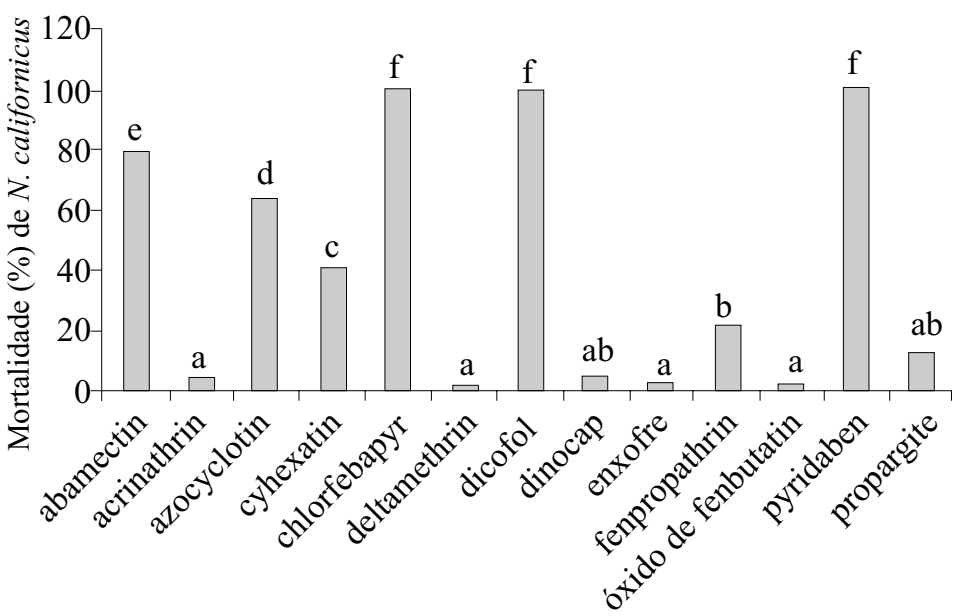

FIGURA 1 - Mortalidade de fêmeas de Neoseiulus californicus, avaliada 72 horas após a aplicação dos produtos. (Colunas com mesma letra não diferem estatisticamente entre si, ao nível de 5\%, pelo teste Tukey).

Dos produtos testados, acrinathrin, azocyclotin, deltamethrin e óxido de fenbutatin não afetaram a reprodução de $N$. californicus, apresentando $E_{R}=1$. Os produtos cyhexatin, dinocap e enxofre foram ligeiramente inferiores a 1 , o que significa um reduzido efeito. Abamectin, fenpropathrin e propargite proporcionaram efeito mais acentuado na reprodução do predador $\left(\mathrm{E}_{\mathrm{r}}=0,760\right.$ a 0,858$)$, comparado ao efeito causado pelos anteriores, pois, quanto menor o valor de $\mathrm{E}_{\mathrm{r}}$, maior o efeito do produto sobre a reprodução (Tabela 2).

Dada a alta mortalidade de fêmeas de $N$. californicus acarretada pelo clorfenapyr, dicofol e pyridaben (100\%), não foi possível avaliar o efeito destes produtos sobre a reprodução do acarino.

A população de $N$. californicus mostrou-se tolerante ao óxido de fenbutatin, comportamento este também constatado para outras espécies de fitoseídeos (Reis \& Sousa, 2001; Reis et al., 1998; Moraes \& McMurtry, 1981). O dinocap não afetou o predador, mostrando baixa sensibilidade do ácaro ao ingrediente ativo, razão pela qual foi classificado como inócuo.
Embora os piretróides, de modo geral, apresentem alta toxicidade aos ácaros fitoseídeos (Reis et al., 1998; Sato et al., 1996; Yamamoto et al., 1992; Zacharda \& Hlùchy, 1991), os produtos acrinathrin, deltamethrin e fenpropathrin, testados no presente trabalho, mostraram-se pouco tóxicos ao $N$. californicus. Easterbrook et al. (2001) constataram, também, baixa sensibilidade do predador $N$. californicus aos piretróides, o que pode indicar uma tolerância natural da espécie a este grupo de produtos.

N. californicus mostrou baixa sensibilidade ao propargite e ao enxofre, o que sugere resistência a estes ingredientes ativos, provavelmente desenvolvida no campo onde foi coletado (morangueiro), uma vez que estes ingredientes ativos são freqüentemente utilizados nesta cultura ou, então, devido a uma tolerância natural. Entretanto, Reis et al. (1998), Sato et al. (1996) e Morse et al. (1987) verificaram alta toxicidade a outras espécies de fitoseídeos com o propargite, e Reis et al. (1999), com o enxofre.

Dos 13 produtos testados, $46 \%$ pertencem à classe 1 (inócuo), $23 \%$ à classe 2 (levemente nocivo), $8 \%$ à classe 3 (moderadamente nocivo) e $23 \%$ à classe 4 (nocivo). Verifica-se que $69 \%$ dos produtos se enquadram nas categorias 1 e 2, ou seja, inócuos e levemente nocivos, portanto podendo ser utilizados; os da classe 3 , somente em condições especiais; e os da classe 4, altamente tóxicos ao $N$. californicus, não devem ser utilizados na cultura quando da presença do fitoseídeo ( Figura 2).

Os resultados obtidos, complementados com trabalhos de campo, contribuirão para uma escolha mais adequada dos agrotóxicos a serem utilizados em programas de manejo integrado, em culturas de citros, nas quais esteja presente $N$. californicus, ou naquelas em que venha a ser liberado. A manutenção deste fitoseídeo nos pomares cítricos pode propiciar maior pressão de controle sobre as populações

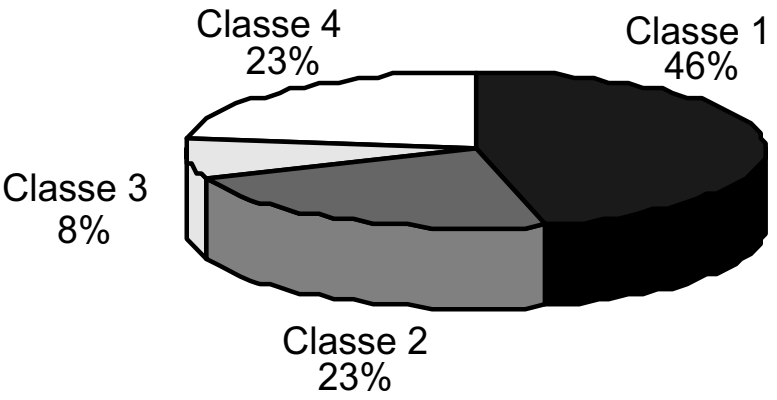

FIGURA 2 - Distribuição dos agrotóxicos testados segundo as classes de toxicidade apresentada à Neoseiulus californicus, conforme IOBC/WPRS: classe 1=inócuo; classe $2=$ levemente nocivo; classe $3=$ =moderadamente nocivo, e classe $4=$ nocivo. 
dos ácaros fitófagos, minimizando as aplicações de acaricidas e reduzindo o custo final da produção.

\section{CONCLUSÕES}

1) Dicofol, pyridaben e chlorfenapyr (classe 4) mostraram-se altamente tóxicos à população de $N$. californicus.

2) Por serem seletivos (classe 1), acrinathrin, deltamethrin, dinocap, enxofre, óxido de fenbutatin e propargite podem ser utilizados em programas de manejo integrado na citricultura, quando se considerar como predador-alvo N. californicus.

3) Azocyclotin, cyhexatin e fenpropathrin ( Classe 2 ) poderão ser utilizados em citros visando ao controle de ácaros fitófagos, na impossibilidade do emprego de produtos de classe 1.

\section{REFERÊNCIAS}

ANDREI, E. (Ed.) Compêndio de defensivos agrícolas. 6. ed. São Paulo: Andrei. 1999. 672p

ARAÚJO, W.F.; MORAES, G.J.de; MERGULHÃO, S.M.R. Seletividade de agroquímicos a ácaros predadores em citros. Petrolina: EMBRAPA-CPATSA, 1989, p.2.

BAKKER, F.M.; GROVE, A.; BLUMEL, S.; CALIS, J.; OOMEN, P. Side-effect test for phytoseiids and their rearing methods. IOBC/ WPRS Bulletin, Montfavest, v.15, n.3, p.61-81, 1992.

BUSOLI, A. C. Uso de enxofre em citros e dinâmica populacional de cochonilhas e ácaros. Laranja , Cordeirópolis, v.13, n.1, p.353395, 1992.

EASTERBROOK, M.A.; FITZGERALD, J.D.; SOLOMON, M.G.; Biological control of strawberry tarsonemid mite Phytonemus pallidus and two-spotted spider mite Tetranychus urticae on strawberry in the UK using species of Neoseiulus (Amblyseius) (Acari: Phytoseiidae). Experimental Applied Acarology, Amsterdam, v.25, n.1, p.25-27, 2001.

HASSAN, S.A.; BIGLE, F.; BLAISINGER, P.; BOGENSHUTZ, H.; BRUN, J.; CHIVERTON, P.; DICKLER, E.; EASTERBROOK, M.A.; EDWARDS, P.J.; ENGLERT, W.D.; FIRTH, S.I.; HUANG, P.; INGLESFIELD, D.; KLINGAUF, F.; KUHNER, C.; OVERMEER, W.P.J.; PLEVOETS, P.; REBOULET, J.N.; RIECKMANN, W.; SAMSOE-PETERSEN, L.; SHIRES, S.W.; STAUBLI, A.; STEVENSON, J.; TUSET, J.J.; VANWETSWINKEL, G.; VANZON, Q. Standard method to test the side-effects of pesticides on natural enemies of insects and mites developed by the IOBC/WPRS working group "Pesticides and Beneficial Organisms". EPPO Bulletin, Oxford, v.15, p.214-255, 1985.

HASSAN, S. A.; BIGLE, F.; BOGENSCHUTZ, H.; BOLLER,E.; BRUN, J.; CHIVERTON, P.; EDWARDS, P.J.; MANSOUR, F.; NATON, E.; OOMEN, P.A.; OVERMEER, W.P.J.; POLGAR, L.; RIECKMANN, W.; SAMSOE-PETERSEN, L.; STAUBLI, A.; STERK,G.; TAVARES, K.; TUSET, J.J.; VIGGIANI, G.; VIVAS, A.G. Result of the sixth joint pesticide testing programme of fhe IOBC/ WPRS - working group "Pesticides and Beneficial Organisms". Entomophaga, Paris, v.39, n.1, p.107-119, 1994.

HENDERSON, C. F.; TILTON, E. W. Tests with acaricides against the brown wheat mite. Journal Economic Entomology, Lanham v.63, p.1536- 1539, 1955.

KNIGHT, A.L.; BEERS, E.H.; HOYT, S.C.; RIEDL, H. Acaricide bioassay with spider mites (Acari: Tetranychidae) on pome fruits: evaluation of methods and selection of discrimination concentrations for resistance monitoring. Journal Economic Entomology, Lanham, v.83, n.5, p.1752-1760, 1990.

KOSTIAINEN, T.; HOY, M.A. The Phytoseiidae as biological control agents of pest mites and insects: a bibliography. Gainesville: University of Florida, 1996. p.355.

McMURTRY, J.A.; CROFT, B.A. Life styles of phytoseiid mites and their roles as biological control agents. Annual Review of
Entomology, Palo Alto, v.42,p.291-321, 1997.

McMURTRY, J.A.; HUFFAKER, C.B.; VAN DE VRIE, M. Ecology of tetranychid mites and their narural enemies: a review. I. Tetranychid enemies: their biological characters and the impact of spray practices. Hilgardia, Berkeley, v.40, n.11, p.331-390, 1970.

MONTEIRO, L.B. Manejo integrado de Panonychus ulmi em macieira. Primeiras experiências com a introdução de Neoseiulus californicus. Revista Brasileira de Fruticultura, Jaboticabal, v.16, p.46-53, 1994.

MORAES, G.J.de. Perspectivas para uso de predadores no controle de ácaros fitófagos no Brasil. Pesquisa Agropecuária Brasileira, Brasília, v.27, p.263-270, 1992.

MORAES, G.J.de; McMURTRY, J.A. Biology of Amblyseius citrifolius (Denmark \& Muma) (Acari: Phytoseiidae). Hilgardia, Berkeley, v.49, n.1, p.1-29, 1981.

MORSE, J.G.; BELLOWS JR., T.S.; GASTON, L..K.; IWATA; Y. Residual toxicity of acaricides to three beneficial species on California citrus. Journal Economic Entomology. Lanham, v.80, p.953-960, 1987.

OMOTO, C.; ALVES, E.B.; RIBEIRO, P.C. Detecção e monitoramento da resistência de Brevipalpus phoenicis (Geijskes) (Acari: Tenuipalpidae) ao dicofol. Anais da Sociedade Entomológica do Brasil, Itabuna, v.29, n.4, p.757-764, 2000.

OVERMEER, W.P.J. Laboratory method for testing side-effects of pesticides on the predaceous mite Typhlodromalus pyri and Amblyseius potentillae (Acari: Phytoseiidae). IOBC/WPRS Bulletin, Montfovet, v.11, n.4, p. 65-69, 1988.

REIS, P.R.; CHIAVEGATO.L.G; MORAES, GJ.de;ALVES, E.B.; SOUSA, E.O. Seletividade de agroquímicos ao ácaro predador Iphiseiodes zuluagai Denmark \& Muma (Acari: Phytoseiidae). Anais da Sociedade Entomológica do Brasil, Itabuna, v.27, n.2, p.265-274. 1998.

REIS, P.R.; SOUSA, E.O.; ALVES, E.B. Seletividade de produtos fitossanitários ao ácaro predador Euseius alatus De Leon( Acari: Phytoseiidae). Revista Brasileira de Fruticultura, Jaboticabal, v.21, n.3, p.350-355,1999.

REIS, P.R.; SOUSA, E.O. Seletividade de chlorfenapyr e fenbutatin-oxide sobre duas espécies de ácaros predadores (Acari: Phytoseiidae) em citros. Revista Brasileira de Fruticultura, Jaboticabal, v.23, n.3, p. 584-588, 2001.

SANTOS, A.C.; GRAVENA, S. Seletividade de acaricidas a insetos e ácaros predadores em citros. Anais da Sociedade Entomológica do Brasil, Itabuna, v.26, n.1, p.99-105,1997.

SATO, M.E.; SILVA, M. da; GONÇALVES, L.R.; SOUZA FILHO, M.F. de; RAGA, A. Toxicidade diferencial de agroquímicos a Neoseiulus californicus (McGregor) (Acari: Phytoseiidae) e Tetranychus urticae Koch (Acari: Tetranychidae) em morangueiro. Neotropical Entomology, Londrina, v.31, n.3, p.449-456, 2002.

SATO, M.E.; RAGA, A.; CERÁVOLO, L.C.; ROSSI, A.C.; POTENZA, M.R. Ácaros predadores em pomar cítrico de Presidente Prudente, Estado de São Paulo. Anais da Sociedade Entomológica do Brasil, Itabuna, v. 23, n.3, p.435-441, 1994.

SATO, M.E.; RAGA, A.; CERÁVOLO, L.C.; ROSSI, A.C.; SOUZA FILHO, M.F. de. Toxicidade residual de acaricidas a Iphiseiodes zuluagai Denmark \& Muma, 1972 (Acari: Phytoseiidae). Arquivos do Instituto Biológico, São Paulo, v.63, p.15-19, 1996.

VAN DE VRIE, M.; McMURTRY, J.A.; HUFFAKER, C.B. Ecology of tetranychid mites and their natural enemies: A review. III. Biology, ecology, and pest status, and host-plant relations of tetranychids. Hilgardia, Berkeley, v.41, p.387-403, 1972.

YAMAMOTO, P.T.; PINTO, A.S.; PAIVA, P.E.B.; GRAVENA, S. Seletividade de agrotóxicos aos inimigos naturais de pragas dos citros. Laranja, Cordeirópolis, v.13, p.709-755, 1992.

ZACHARDA, M.; HLÙCHY, M. Long-term residual efficacy of commercial formulations of pesticides to Typhlodromus pyri Scheuten (Acari: Phytoseiidae) inhabiting commercial vineyards. Experimental and Applied Acarology, Amsterdam v.13, p.27-40, 1991. 